\title{
DETERMINATION OF SIZE SPARK DISCHARGE BETWEEN ELECTRODES AT ELECTROSPARK PROCESSING DETAILS OF AGRICULTURAL MACHINES
}

\section{РАСЧЕТ РАЗМЕРА ИСКРОВЫХ РАЗРЯДОВ ПРИ ЭЛЕКТРОИСКРОВОЙ ОБРАБОТКЕ ДЕТАЛЕЙ СЕЛЬСКОХОЗЯЙСТВЕННЫХ МАШИН}

\section{Kuznetsov, Post-graduate student \\ И.С. Кузнецов, аспирант}

\section{Pavlov, Candidate of Technical Sciences, Associate Professor}

B.3. Павлов, кандидат технических наук, доцент

A. Kolomeichenko, Doctor of Technical Sciences, Professor

А.В. Коломейченко, доктор технических наук, профессор

Orel State Agrarian University, Orel City, Russia

Орловский государственный аграрный университет, г. Орёл, Россия Phone: +7 (4862) 43-19-79, E-mail: ivan-654@yandex.ru

Received June 13, 2012

\begin{abstract}
The paper implemented the model calculation of spark discharge diameter, presented in form of dependency on diameter of cross sectional area of electrodes, voltage and parameters of gaseous medium in which electrospark processing. Determined ratio of estimated coefficient due to stress and average distance between electrode and workpiece (anode - electrode, cathode - detail).
\end{abstract}

\section{АННОТАЦИЯ}

В работе представлена модель определения диаметра искрового разряда, в зависимости от площсади сечения электродов, напряжения и параметров газовой среды,, в которой проводится электроискровая обработка. Определен необходимый для расчета коэффициент, обусловленный отношением напряжения и средним расстоянием между электродом и деталью (анод электрод; катод - деталь).

\section{KEY WORDS}

Electrospark processing; Spark discharge; Diameter; Dissociation energy; Ionization energy; Crosssectional area; Electrodes.

\section{КЛЮЧЕВЫЕ СЛОВА}

Электроискровая обработка; Искровой разряд; Диаметр; Энергия диссочиаџии; Энергия ионизачии; Площадь сечения; Электродыл.

Диаметр канала искрового разряда определяет на электродах размер пятна разогрева их поверхностей. Вместе с величиной кинетической энергии столкновения заряженных частиц (электронов и ионов) с поверхностями электродов размер пятна разогрева за время сближения электродов позволит рассчитать интенсивность тепловых источников на поверхностях, количество поступающей теплоты и температурные поля в электродах.

Диаметр канала искрового разряда после его возникновения определяется вели- чиной тока между электродами, которая зависит от затрат электрической энергии на диссоциацию и ионизацию молекул воздуха, на ускорение заряженных частиц в электрическом поле.

Энергия диссоциации молекул азота рассчитывается по формуле:

$$
W_{N}=0,78 \cdot n e V_{N}
$$

где $W_{N}$ - энергия диссоциации молекул азота, Дж/м ${ }^{3} ; n-$ число двухатомных молекул воздуха, $1 / \mathrm{M}^{3} ; e-$ заряд электрона, $\kappa л ; V_{N}-$ 
потенциал диссоциации молекул азота; 0,78 - коэффициент учитывающий объемную долю азота.

Количество молекул воздуха в единице объема:

$$
n=\frac{P_{0}}{k T_{0}}
$$

где $\mathrm{P}_{0}$ - начальное давление среды, Па; $\mathrm{k}$ постоянная Больцмана, Дж/ ${ }^{\circ} \mathrm{K} ; \mathrm{T}_{0}-$ начальная температура среды, ${ }^{\circ} \mathrm{K}$.

Энергия диссоциации молекул кислорода:

$$
W_{N}=0,21 \cdot n e V_{0}
$$

где $\mathrm{W}_{0}$ - энергия диссоциации молекул кислорода, Дж/м ${ }^{3} ; V_{0}-$ потенциал ионизации молекул кислорода, эВ; 0,21 - коэффициент учитывающий объемную долю кислорода.

При одинарной ионизации количество образовавшихся ионов составляет:

$$
N_{i}=2 n,
$$

и равно количеству электронов $N_{e}$. Потенциалы ионизации азота и кислорода отличаются незначительно и в оценочном расчете с учетом большей объемной доли азота могут быть приняты равными:

$$
V_{u}=14,2, \text { э } B
$$

Энергия ионизации атомов воздуха при пробое пространства между электродами:

$$
W_{u}=2 n e V_{u},(4)
$$

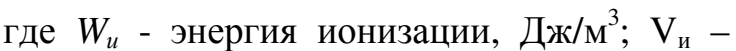
потенциал ионизации, эВ.

Под действием сил электрического поля заряженные частицы ускоряются и приобретают преобладающее направление движения вдоль поля. Осредненное движение электронов и ионов происходит с некоторыми средними скоростями дрейфа, величина которых зависит от размеров и массы частиц, напряжения между электродами [1].

Скорость дрейфа определяет среднюю кинетическую энергию ионов и электронов, полученную за счет энергии электрического поля между электродами:

$$
W_{\ni}=\frac{1}{2}\left(m_{e} N_{e} V_{d e}^{2}+m_{i} N_{i} V_{d i}^{2}\right)
$$

где $W_{\text {э }}$ - расход энергии поля на ускорения частиц до скоростей дрейфа, Дж/м ${ }^{3} ; m_{e} u m_{i}$
- масса электрона и иона соответственно, кг; $V_{d e}$ и $V_{d i}$ - средняя скорость дрейфа электронов и ионов, соответственно, м/с.

С учетом оценочных величин скоростей дрейфа $V_{d i}$ и $V_{d e}$, рассмотренных авторами в предыдущей работе (О движении заряженных частиц между электродами при электроискровой обработке), формула (5) примет вид:

$$
W_{\ni}=\frac{e E_{n p}}{2 \pi r_{i}^{2}}
$$

где $\mathrm{E}_{\text {пр }}$ - напряжение пробоя, $\mathrm{B} / \mathrm{M} ; \mathrm{r}_{\mathrm{i}}$ - радиус иона, м.

Энергия разряда между электродами затрачивается на диссоциацию молекул, ионизацию атомов и на увеличение кинетической энергии заряженных частиц (нагрев газа):

$$
W=W_{N}+W_{0}+W_{u}+W_{\ni}
$$

где $W$ - энергия разряда, Дж/м ${ }^{3}$. Общая энергия разряда соответствует конденсатору, образованному электродами:

$$
W=\frac{C U^{2}}{2},
$$

где $C$ - емкость конденсатора, $\Phi$; $U$ - напряжение между электродами, В. Емкость конденсатора, образованного электродами:

$$
C=\varepsilon_{0} \frac{S}{h}
$$

где $\varepsilon_{0}$ - диэлектрическая постоянная, л$^{2} / \mathrm{H} \cdot \mathrm{m}^{2}$; $S$ - площадь сечения электродов (анода), м $^{2}$; $h$ - расстояние между электродами, м.

Переменное расстояние между электродами [2]:

$$
h(\tau)=A\left[1-\sin 2 \pi f\left(\tau+\tau_{c}\right)\right],
$$

где $A$ - амплитуда колебаний вибратора, м; $f$ - частота, Гц; $\tau$ - время, с; $\tau_{c}-$ время наступления пробоя, с.

Среднее расстояние $h_{c p}$ за время сближения $\tau_{\ni}$ электродов:

$$
\begin{aligned}
& h_{c p}=\frac{A}{\tau_{\ni}} \int_{0}^{\tau_{\ni}}\left[1-\sin 2 \pi f\left(\tau+\tau_{c}\right)\right] d \tau= \\
& =A\left[1-\frac{1}{\pi f \tau_{\ni}} \cdot \sin \pi f\left(\tau_{\ni}+2 \tau_{C}\right) \cdot \sin \pi f \tau_{\ni}\right]
\end{aligned}
$$


Учитывая, что по порядку величины $\sin \pi f \tau_{\ni} \approx \pi f \tau_{\ni}$, получим:

$$
h_{c p} \approx A\left[1-\sin \pi f\left(\tau_{\ni}+2 \tau_{C}\right)\right]
$$

и средняя величина емкости конденсатора, образованного электродами, за время их сближения составит:

$$
C_{c p}=\varepsilon_{0} \frac{S}{h_{c p}}
$$

Средние скорости дрейфа ионов и электронов определяют только направленную вдоль поля составляющую полной скорости частиц. Другая составляющая полной скорости частиц - это движение зарядов поперек поля (боковая скорость). Эта поперечная скорость определяется разностью давления в канале разряда и окружающей среде, которая приводит к снижению объемной плотности заряженных частиц в разряде и, в конечном счете, к разрушению разряда с последующим образованием нового разряда за счет притока воздуха окружающей среды.

Примем, что за время между разрядами энергия конденсатора, образованного электродами, успевает восстанавливаться за счет энергии вибратора (генератора). Полная потеря электрической энергии происходит только при контакте электродов. Тогда в формуле (8) можно пренебречь падением напряжения и принять $\mathrm{U}=\mathrm{U}_{0},\left(\mathrm{U}_{0}-\right.$ начальное напряжение генератора, подаваемое на электроды).

Тогда общая энергия разряда между электродами:

$$
W=\frac{\varepsilon_{0} S}{2 h_{c p}} \cdot U_{0}^{2}
$$

Уравнение баланса энергии в разряде (7) примет вид:

$$
\frac{\varepsilon_{0} S U_{0}^{2}}{2 h_{c p}}=\frac{\pi d^{2}}{4} h_{c p}\left(W_{N}+W_{0}+W_{u}+W_{\ni}\right)
$$

Откуда, с учетом равенств (1), (3), (4), (6) найдем средний диаметр разряда:

$$
d=\frac{U_{0}}{h_{c p}} \sqrt{\frac{2 \varepsilon_{0} S}{\pi e B}}
$$

где $B$ - коэффициент, который определяется:

$$
B=n\left(0,78 \cdot V_{N}+0,21 V_{0}+2 V_{u}\right)+\frac{E_{n p}}{2 \pi r_{i}^{2}}
$$

Отношение:

$$
\frac{U_{0}}{h_{c p}}=\frac{U_{0}}{A\left[1-\sin \pi f\left(\tau_{\ni}+2 \tau_{C}\right)\right]}
$$

Разряд возникает при сближении электродов до расстояния пробоя. Очевидно, что диаметр разряда не должен зависеть от амплитуды колебаний вибратора. Значит при изменении амплитуды колебаний остальные величины в отношении (18) должны изменяться соответствующим образом, согласуясь с изменением амплитуды. На рисунке 1 представлены результаты расчета отношения $U_{0} / h_{c p}$ по формуле (18).

Как видно из графика, можно принять:

$$
\frac{U_{0}}{h_{c p}}=1,27 \cdot 10^{7}, B / \mathcal{M}
$$

Тогда расчетная зависимость для диаметра разряда примет вид:

$$
d=1,27 \cdot 10^{7} \sqrt{\frac{2 \varepsilon_{0} S}{\pi e B}}, \mu
$$

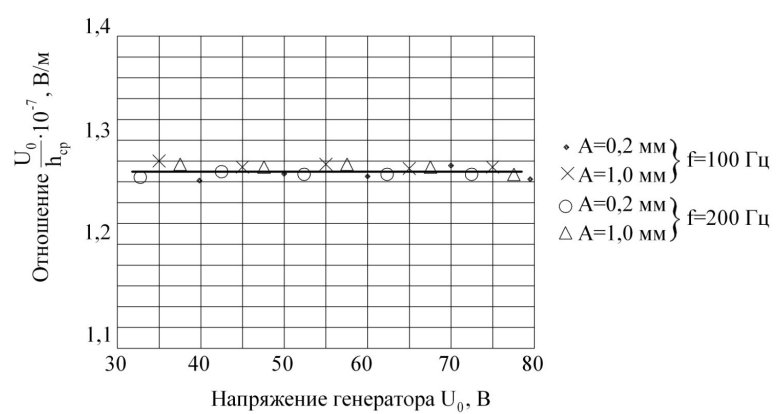

Рисунок 1 - Результаты расчета отношения $\frac{U_{0}}{h_{c p}}$

Полученная зависимость может служить для предварительного расчета размеров искрового разряда, так как не учитывалось некоторое снижение напряжения за время существования каждого единичного разряда. Поэтому её необходимо будет уточнять после определения длительности каждого единичного разряда при сближении электродов.

\section{БИБЛИОГРАФИЯ}

Фейнман, Р. Фейнмановские лекции по физике: Кинетика. Теплота. Звук. Пер. с англ. Т.4.[Текст] / Р. Фейнман, Р. Лейтон, М. Сэндс // М.; Эдиториал УРСС., - 2004. 264 c. 